\title{
Telecommunications on Earth and their possible relation with the anthropic principle
}

\section{IGNACIO DEL VILLAR ${ }^{1,2}$}

${ }^{1}$ Department of Electrical and Electronic Engineering, Public University of Navarre, 31006 Pamplona, Spain

${ }^{2}$ Institute of Smart Cities, Public University of Navarre, 31006 Pamplona, Spain ignacio.delvillar@unavarra.es ORCID: 0000-0002-8130-4035

\begin{abstract}
The society of the information is based on the ability of humans to communicate in real time. This is possible thanks to the emission of electromagnetic waves. In this work it is discussed, by analyzing the round trip time (RTT) parameter, if it is possible to expand the society of the information out of the Earth. The results show that the value of the speed of light limits this expansion. Real time applications, such as videogaming, remote surgery, or voice conversations, are only possible on Earth and its proximities, which indicates that there could be a relation between real time telecommunications on Earth and the anthropic principle.
\end{abstract}

Keywords: anthropic principle; communications; round trip time; delay; speed of light. 


\section{Introduction}

Human beings communicate with each other in two different ways. The first way is the same used by other animals: the emission of sound waves. However, sound waves emitted by a human being typically propagate no more than a few tens of meters if there are no special conditions like echoes in the mountains. The use of a microphone could solve this issue, but nobody can imagine a conversation between two persons, one of each with a microphone, separated more than one hundred meters. This makes no sense because, in addition, all other people in the perimeter would hear the conversation and probably they are not interested in it. If this method was extended to many conversations in the same town, life would become a complete chaos with many conversations that are heard by every people. Nature is designed in terms of sound wave communication for another purpose, for communicating people at short distances without being all the people in the surroundings affected by these conversations; in other words, for face to face social relations.

However, sometimes we do not have the opportunity to see the person we want to talk to. Many centuries ago this was a big limitation. Somebody travelled abroad and the only way to communicate was by letter, with a delay of the order of days, or even months. The human being improved this technology a lot in terms of guarantee of delivery. For example, it is amazing how the Spanish king could manage to send orders to viceroys in America, though the missive took a very long time to arrive at its destination. The famous book of Jules Verne, “The Courier of the Czar", was also based on the concept of sending information surely and efficiently, but with the limitation of a very long delay.

There existed alternatives, such as smoke signals or flags. However, though faster than a letter, they were very inefficient in terms of the amount of information that could be transmitted. An important improvement was made in 1791 by Claude Chappe, the inventor of the optical telegraph, a system which consisted in a network of stations that allowed the transmission of information at a speed of 1 symbol every two minutes between Paris and 
Lille (i.e. $230 \mathrm{~km}$ ) (Dilhac 2001). Each station monitored, with the aid of a telescope, the character that was represented with a wooden semaphore in the previous station. However, the system depended on weather conditions (the presence of rain or fog disturbed the communication) and obviously it did not work during the night.

The previous issues were solved in the nineteenth century, thanks to one of the most important milestones in the history of science and technology: the implementation of the first electric telegraph. Many scientists and engineers contributed to the success of this method of communication, but perhaps the most important achievement occurred in 1837, with the connection of two train stations in England, Euston and Camden Town, which were separated from each other by $2 \mathrm{~km}$. The inventors were William F. Cooke and Charles Wheatstone (Black 1983). In very few years is was possible to communicate the United States from west to east, and later on it became a reality to achieve transoceanic communication with submarine cables. All this refers to electromagnetic waves guided through a wire. Later on, in 1895, Guglielmo Marconi started to develop the first experiments on wireless telegraphy, which led to the first wireless communication across the Atlantic Ocean (Falciasecca and Valotti 2009). During the twentieth and twenty first century both guided and wireless communications at long distance were improved thanks to the application of optical fiber and modern wireless technology, leading to the creation of the society of the information, where each person is interconnected with the other in real time. Nonetheless, everything had already started in the $19^{\text {th }}$ century, with the successful propagation of guided and wireless electromagnetic signals.

Electromagnetic wave based communications is something that makes human beings unique. Unlike other animals, we can emit electromagnetic signals with the aid of machinery designed ad hoc. It is true that there exist animals such as the electric fish that communicate with each other via electric signals (Theodore Holmes Bullock 1973; Hagedorn and Heiligenberg 1985), but this is possible because they include this system of communication in their own body. The human being, despite the disadvantage that has no internal apparatus for electric communication with other people, has 
overcome this issue by designing machines that can emit electromagnetic signals. Moreover, unlike for the case of electromagnetic signals emitted by animals, the human being has fabricated equipment that regenerates the signal when after some distance it is attenuated. In this way, electromagnetic signals can be transmitted at distances of thousands of kilometers. There are two types of machines that allow regenerating the signal: amplifiers and repeaters, which present some differences but which in general are focused on allowing the electromagnetic signal being propagated through hundreds of kilometers up to a limit that all points at the earth surface can be interconnected. This is one of the greatest milestones in the history of science.

However, one question arises. Why have we focused on electromagnetic waves, which we cannot emit with our body, and not on sound waves, towards the construction of the information society?

\section{The round trip time: the parameter that defines the quality of communications}

As stated above, human beings can communicate with each other through sound waves. Unlike for electromagnetic wave based communication, with sound waves there is an evident limitation in terms of distance between the interlocutors. There are two basic elements that prevent a sound wave based telecommunication between human beings to take place. The first one is the attenuation of the signal. Attenuation is a common issue in all types of communication. However, as stated above, repeaters and amplifiers have been designed to counteract the attenuation effect in the domain of electromagnetic waves. Why not in the case of sound waves? All the engineering and scientific effort that has been made towards propagation of electromagnetic signals towards interconnecting the entire world could have been devoted to the propagation of sound waves. But this is not a reality because of the second element that hinders sound wave based communication: the propagation delay. Propagation delay is the time a signal takes to be transmitted from one point to another one and it can be obtained with a very simple equation: 


$$
t_{p}=\frac{d}{v_{p}}
$$

where $t_{p}$ is the propagation time, $v_{p}$ is the velocity of propagation and $d$ is the distance between the emitter and the receiver.

If we consider that the speed of sound in air is approximately $340 \mathrm{~m} / \mathrm{s}$, we will easily deduct that it takes circa three seconds to transmit a voice signal between an sender and a receiver that are separated $1 \mathrm{~km}$ from each other. Three seconds is a huge amount of time in a world where communications are nearly instantaneous, and this delay is only for $1 \mathrm{~km}$. If the distance was $1000 \mathrm{~km}$ it would take 3000 seconds, nearly one hour to transmit the signal. This explains why sound waves are not used for global communications. Oppositely, electromagnetic waves propagate at velocities of about $3 \times 10^{8}$ $\mathrm{m} / \mathrm{s}$, which allows communicating nearly immediately between any two points of the earth. This justifies the success of this type of communication. However, in order to have a better knowledge of both sound wave and electromagnetic wave based communications it is necessary to define some more basic concepts.

There is a parameter that gives a better knowledge on the quality of communications: the round trip time (RTT). The RTT, also called ping time, is usually employed as a parameter that indicates the quality of a communication and it is closely related to the propagation delay. It is basically the time it takes a sender to transmit a signal and receive an answer for the receiver. This can be shown schematically in Fig. 1.

The RTT can be obtained with this equation:

$$
R T T=2 \times \text { paquet delivery time }+ \text { processing delay }
$$

The processing delay is the time the sender and receiver spend in processing data, whereas the packet delivery time is:

$$
\text { paquet delivery time }=\text { transmission time }+ \text { propagation delay }
$$




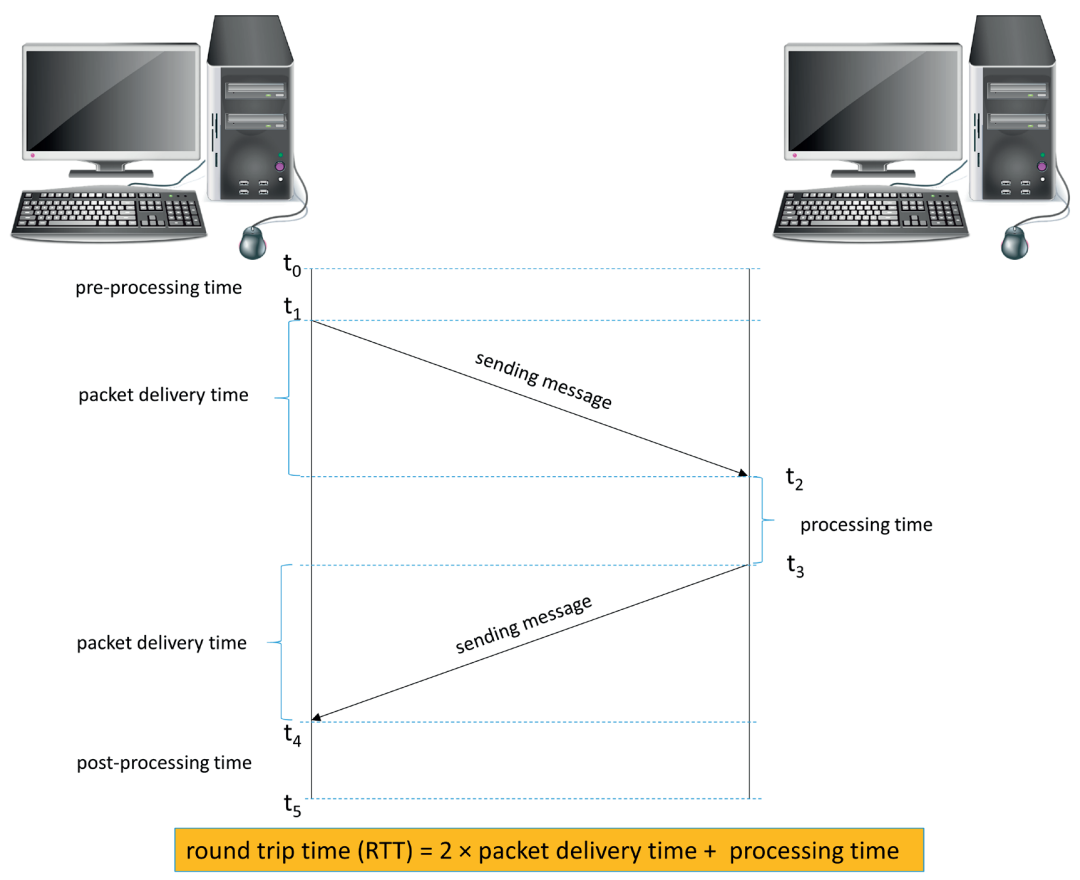

Figure 1. Schematic explanation of the round trip time (RTT)

The propagation delay was explained above, whereas the transmission time is:

$$
\text { transmission time }=\frac{\text { number of bits transmitted }}{\text { bit rate }}
$$

where the bit rate is the number of bits per second transmitted by the sender Obtaining the RTT seems to be a complex calculation after all these equations, but it can be greatly simplified if the propagation delay is much higher than the processing delay (by improving the processing speed) and the transmission time (by improving the bit rate). In such situations, equation (2) can expressed as: 
or in terms of distance $d$ and velocity of propagation $v p$ :

$$
R T T \sim \frac{2 \times d}{v_{p}}
$$

Engineers and scientists define values around $200 \mathrm{~ms}$ as a threshold for quality in communication. This is based on typical round trip times considered acceptable in several applications. Game designers try to optimize for 200 to $250 \mathrm{~ms}$ the round trip time (Färber 2002), because for higher values than $200 \mathrm{~ms}$ delays become noticeable. Eye gaze prediction systems also can operate adequately with RTT values below $200 \mathrm{~ms}$ (Yunlong Feng Gene Cheung 2013). Regarding telephone service, a delay of $150 \mathrm{~ms}$ in one direction is the limit accepted in terms of quality, what means an $\mathrm{RTT}=150 \mathrm{x} 2=300 \mathrm{~ms}$ (Kassim et al. 2017). Finally, remote surgery is another important field where surgeons can work with RTT of $300 \mathrm{~ms}$ (Lum et al. 2009; Lester and Thronson 2011). In view of these examples, where the adequate RTT is 200-300 ms, we will consider the more demanding case, $200 \mathrm{~ms}$, which should satisfy the needs for most of telecommunication applications. So we will consider henceforward as general value that the RTT must not exceed $200 \mathrm{~ms}$ in order to implement long distance real time applications.

\section{The limits of sound and electromagnetic wave based communications}

Considering that the speed of sound is $340 \mathrm{~m} / \mathrm{s}$, and that the RTT should not exceed $200 \mathrm{~ms}$, from expression (6) we can obtain:

$$
200 m s>\frac{d \times 2}{340 m / s}
$$


From the previous expression it can be concluded that the distance for a conversation between two persons should not exceed 34 meters. Normal conversations do not exceed this distance because, on the other hand, for such a long distance one needs to shout, and even though amplifiers could be used, it would become a complete chaos because all people would hear all conversations and would be disturbed, as stated in the introduction. But even if we could face this problem, the basic difficulty is that the velocity of propagation is very low and real time communication is only possible at a very short distance of tens of meters.

Regarding electromagnetic signals, they can be propagated nowadays through both guided and wireless media. It is well known that the velocity of propagation in vacuum is $3 \times 10^{8} \mathrm{~m} / \mathrm{s}$. However, this value is reduced when transmission is done through matter, due to absorption and reemission of the wave energy by the atoms of the material. That is why engineers use a parameter that is called velocity factor, which is the percentage of the maximum velocity of propagation that is achieved. As an example, the velocity of propagation is $2.1 \times 10^{8} \mathrm{~m} / \mathrm{s}$ in a communication medium that has a velocity factor of 0.7 . This reduction can be calculated in different ways depending on the medium of propagation. As an example, for optical fiber the velocity of propagation is:

$$
v_{p}=\frac{c}{n}
$$

where $\mathrm{c}$ is the speed of light in vacuum, $3 \times 10^{8} \mathrm{~m} / \mathrm{s}$, and $n$ is the refractive index of the transmission medium. In view that silica is the most typical medium used for optical communications, and that it has a refractive index whose value is approximately 1.45 , that is why engineers accept that the velocity of propagation in fiber is $2 \times 10^{8} \mathrm{~m} / \mathrm{s}$ (Santos et al. 2012). The other typical guided medium (used in many Ethernet networks) is copper. For this medium the calculation of the velocity of propagation is different because it depends rather on the permittivity copper and the insulator. However, the velocity factor achieved is similar for optimized cables. Consequently, engineers accept that the velocity in copper is approximately $2 \times 10^{8} \mathrm{~m} / \mathrm{s}$ 
(Schalk et al. 2017). Regarding wireless networks, air refractive index is very similar to vacuum refractive index. As a result, $3 \times 10^{8} \mathrm{~m} / \mathrm{s}$ is assumed (Su et al. 2005; Gajjar et al. 2014).

It is true that wireless is used for satellite communications, but its usage is not as widely extended as terrestrial communications. One well-known application is satellite television, where geostationary satellites located at approximately 36,000 $\mathrm{km}$ cover the same regions of the Earth and in this way end users just need to orient their parabolic antennas to the right position in order to receive the signal. However, the distance from the base station on Earth to the receiver is $2 \times 36,000=72,000 \mathrm{~km}$. This distance is nearly double than the separation between the two farthest points on Earth, which causes a delay in the reception of the television signal (Iera, Molinaro, and Marano 2002). This delay is acceptable for this application but the idea could not be extrapolated to the internet, where there is an additional processing time for multiple interactions of end users. The global positioning system (GPS) is another example of wireless communication and it uses satellites that send clock signals that are interpreted by the GPS receiver. The satellites are located at a shorter distance, typically $20,000 \mathrm{~km}$, which allows reducing the delay time, but it must be pointed out that this technology is not interactive; it is a one way communication where the satellites just send the clock signals that are interpreted by the GPS receiver. If we want to reduce the delay time, in such a way that real interactive communications are possible, low earth orbit (LEO) satellites, located at approximately 1,000 km, are preferred (Sanctis et al. 2016). With this technology it is possible to think about satellite internet communications.

Nonetheless, though satellite communications have been improved a lot, terrestrial communications have the advantage of a shorter distance when interconnecting any two points in the global surface, something that has been possible thanks to optical fiber, which especially in long distance communications has no competitor. So only in some exceptional cases, such as planes or remote and small islands, there is a need for satellite communications, whereas in the rest of cases the optical fiber network can be extended to the end user with the aid of a short distance wireless 
connection. Regarding copper, its utilization is being reduced to the local area networks in offices or buildings, and its usage is decaying.

In order to understand the importance of optical fiber in communications, it must be highlighted that it is the only transmission medium used for transoceanic communications: more than 420 undersea optical telecommunications covered a distance of over 1.1 million $\mathrm{km}$ in the year 2017, a network whose length is increasing year after year (Hartog and Clare 2018). That is why for the sake of simplicity the next calculations will be performed on the basis that the communication medium is optical fiber, with a propagation velocity of $2 \times 10^{8} \mathrm{~m} / \mathrm{s}$.

Reconsidering expression (6), we will do the same calculation of expression 7 but for a propagation velocity of $2 \times 10^{8} \mathrm{~m} / \mathrm{s}$.

$$
200 m s>\frac{d \times 2}{2 \times 10^{8} \mathrm{~m} / \mathrm{s}}
$$

In this occasion the distance is not 34 meters but 20,000 km. This indicates that the two farthest points we can have in a conversation on Earth is $20,000 \mathrm{~km}$, and precisely this is the farthest separation between any two points on the surface the Earth (because the circumference of the Earth is $40,000 \mathrm{~km})$.

The speed of propagation of electromagnetic waves is exactly adequate for being able to converse in a fluid way among all the inhabitants of the Earth and it is not adequate, for example, for the Moon (the distance is $384,000 \mathrm{~km}$, much higher than the limit of 20,000 km) and much less for Mars, since the distance is even higher than the distance from the Earth to the Moon.

It is evident that for the calculation of the RTT there are important additional factors such as the processing delay and the transmission time, indicated in expressions (2) and (3), but these values can be reduced by adequate processing to such a point that the key limiting factor is the propagation delay, which basically depends on the propagation velocity of electromagnetic waves and cannot be modified. 
Two main conclusions can be extracted from the previous calculations. The first one is that if the speed of light was lower it would not be possible to communicate any two points on the earth without running risk that the RTT exceeds 200 ms. In other words, worldwide real time communication could not be possible. The information society, which depends on this factor, would collapse. As an example, if the propagation speed of light in fiber was $2 \times 10^{7} \mathrm{~m} / \mathrm{s}$ instead of $2 \times 10^{8} \mathrm{~m} / \mathrm{s}$, the RTT between Buenos Aires and Seoul (separated by nearly 20,000 km) would increase from 200 to 2,000 ms. It would take 2 seconds to acknowledge any data sent from Argentina and South Korea. This would add a lot of uncomfortability in telephone calls, whereas more demanding applications such as remote surgery or interactive videogames could not face this increase of the RTT.

The second conclusion is that in order to have an adequate RTT in interplanetary communications we would need to increase the speed of light, because with $3 \times 10^{8} \mathrm{~m} / \mathrm{s}$ the RTT, even for Earth to Moon communication, is unacceptable. Considering that the distance between the Earth and the Moon is approximately $400,000 \mathrm{~km}$, in order to have an RTT of $200 \mathrm{~ms}$ it would be necessary to increase the speed of light up to $4 \times 10^{9} \mathrm{~m} / \mathrm{s}$, and this increase should even be higher for communications between the Earth an other planets. This is explained because the RTT increases as we increase the distance between the interlocutors. In order to visualize this effect, Fig. 2 shows the RTT for telecommunication between the Earth and the different planets of the solar system. As a reference, a line showing the maximum acceptable RTT for real time applications and the RTT for the two furthest points on Earth is offered.

Many scientists and engineers would long for interplanetary missions avoiding long RTTs, but this is not possible due to the value of the speed of light. This limitation leads to reception of images from Jupiter hours later than they are taken (Sarkar 2011), or conversing in an eventual human mission in Mars with delays of 3 to 22 minutes (the separation between Earth and Mars is changing due to the orbit) (Lester and Thronson 2011). Only in the case of Earth-Moon communication the minimum RTT is 2.6 seconds (Love and Reagan 2013; Lester and Thronson 2011). In view of this 
issue, engineers focus on how to handle communications in conditions of high latency and also of low bandwidth, those that will be present in the exploration of planets in our solar system (Beaton et al. 2017; Chappell et al. 2016). They assume this problem and try to optimize non-real time communications in the best conditions possible (Lester and Thronson 2011).

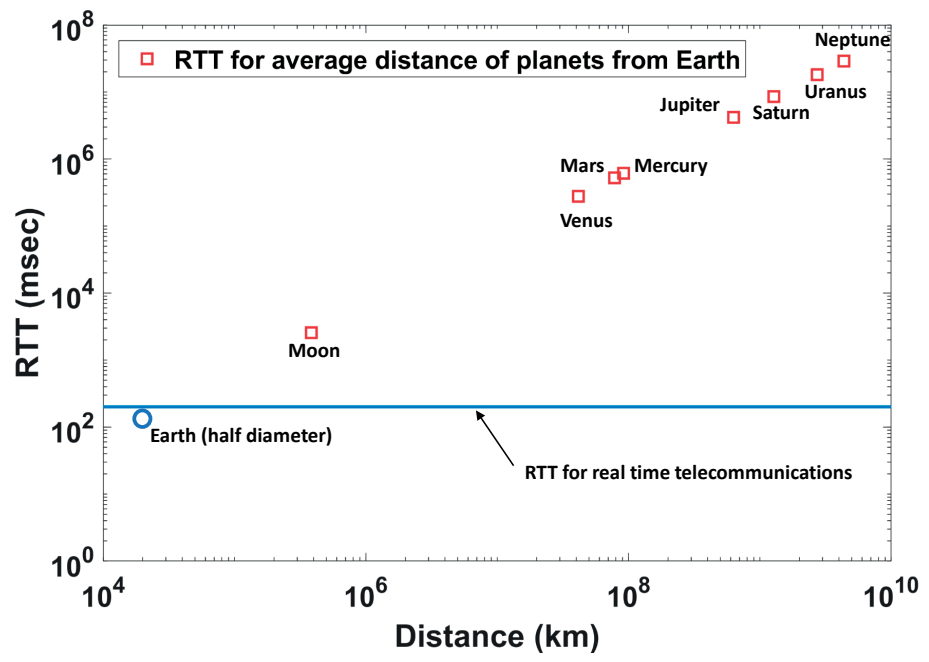

Figure 2. RTT as a function of the average distance of the planets from Earth. As a reference the RTT between the two most separated points on the Earth surface is shown. Also the RTT for real time applications is shown as a threshold value

However, in addition to the technological implications of the fact that the speed of light limits real time applications to the spherical surface or the proximities of the Earth, a philosophical reflection can be made. Global real time communications are only possible in planets not bigger than the Earth, and in addition it is necessary an intelligent animal like the human being, which artificially controls the amplification and propagation of electromagnetic signals (animals can emit electromagnetic signals but it must be pointed out that the best medium of transmission, optical fiber, 
needs amplification typically every $10 \mathrm{~km}$ ). Some people will say that this just a coincidence, whereas others may relate this with the anthropic principle, which asserts that existence of intelligent observers determines the fundamental structure of the Universe (Barrow 1986).

\section{Discussion and conclusion}

The quality of a communication system depends on the round trip time (RTT), which is the time it takes a sender to transmit a signal and receive an answer for the receiver.

It is generally accepted that a maximum RTT of $200 \mathrm{~ms}$ is adequate for most real time telecommunication applications, and it has been observed that this value is precisely the RTT obtained when connecting the two farthest points on the Earth surface. As a result, it is possible to interconnect any two points on the Earth's surface without exceeding the RTT that is adequate for real time applications. However, only moving up to the closest celestial object, the Moon, allows realizing that the RTT increases to 2,600 ms, a value inacceptable for many of the applications we use in our society of the information. For Mars, our closest planet along with Venus, the RTT increases to minutes, a value that continues to grow for Jupiter, Saturn, Uranus and Neptune. Moreover, if the closest star, Proxima Centauri, had a planet, the RTT would be $2 \times 4.2=8.4$ years, because it is separated from our solar system by 4.2 light-years (i.e. the time it takes light to propagate from the Earth to this star).

All this indicates that telecommunications become more difficult in terms of latency as we separate from our planet. Real time telecommunications and, hence, the society of information, is only possible in planets whose diameter is not bigger than the diameter of the Earth, and only an intelligent animal like the human being, capable of controlling the propagation of electromagnetic signals, can benefit from this technology. This paradoxical coincidence opens the path for philosophical questions. One of them is why the evolution of the human being, in terms of time of reaction to events, has converged with the development of the society of the infor- 
mation in a planet like the Earth. The RTT of $200 \mathrm{~ms}$, considered adequate for real time applications, is valid because our brain, combined with other parts of our body like our eyes and ears, reacts to different stimulus with response times that fit this value of the RTT. This RTT is the result of many years of evolution, and the diameter of the Earth has been also the result of the expansion of the universe. The third parameter, the speed of light, combines with the RTT and the diameter of the Earth towards the creation of the society of the information, which basically consists of many human beings interacting with each other in real time on the surface of our planet. A second philosophical question is about the sense of colonizing planets when it is not possible to communicate with them in real time. Will we be able in the future to communicate with each other exceeding the speed of light? Probably the reader of this article will propose other questions that may emerge from the paradoxical coincidence presented in this work.

\section{References}

Barrow, John D. 1986. The Anthropic Cosmological Principle. Oxford University Press. Beaton, Kara H., Steven P. Chappell, Andrew F.J. Abercromby, Matthew J. Miller, Shannon Kobs Nawotniak, Scott S. Hughes, Allyson Brady, and Darlene S.S. Lim. 2017. "Extravehicular Activity Operations Concepts under Communication Latency and Bandwidth Constraints.” In IEEE Aerospace Conference Proceedings, 7943570. https://doi.org/10.1109/AERO.2017.7943570.

Black, Robert Monro. 1983. “Early Telegraph Cables.” In The History of Electric Wires and Cables. London: IET.

Chappell, Steven P., Trevor G. Graff, Kara H. Beaton, Andrew F.J. Abercromby, Christopher Halcon, Matthew J. Miller, and Michael L. Gernhardt. 2016. “NEEMO 18-20: Analog Testing for Mitigation of Communication Latency during Human Space Exploration.” In IEEE Aerospace Conference Proceedings, June:7500717. https://doi.org/10.1109/AERO.2016.7500717.

Dilhac, J-M. 2001. “The Telegraph of Claude Chappe: An Optical Telecommuication Network for the 18th Century.” In IEEE Conf. on the History of Telecommun.

Falciasecca, Gabriele, and Barbara Valotti. 2009. "Guglielmo Marconi: The Pioneer of Wireless Communications.” In European Microwave Week 2009, EuMW 2009: Science, Progress and Quality at Radiofrequencies, Conference Proceedings - 39th 
European Microwave Conference, EuMC 2009, 544-46. https://doi.org/10.1109/ EUMC.2009.5296358.

Färber, Johannes. 2002. "Network Game Traffic Modelling.” In Proceedings of the 1st Workshop on Network and System Support for Games - NETGAMES '02, 53-57. https://doi.org/10.1145/566500.566508.

Gajjar, Sachin, Nilav Choksi, Mohanchur Sarkar, and Kankar Dasgupta. 2014. "Comparative Analysis of Wireless Sensor Network Motes.” 2014 International Conference on Signal Processing and Integrated Networks (SPIN), 426-31. https:// doi.org/10.1109/SPIN.2014.6776991.

Hagedorn, Mary, and Walter Heiligenberg. 1985. "Court and Spark: Electric Signals in the Courtship and Mating of Gymnotoid Fish.” Animal Behaviour 33 (1): 254-65. https://doi.org/10.1016/S0003-3472(85)80139-1.

Hartog, Arthur H, and Michael A Clare. 2018. “Advances in Distributed Fiber-Optic Sensing for Monitoring Marine Infrastructure, Measuring the Deep Ocean , and Quantifying the Risks Posed by Sea Fl Oor Hazards” 52 (5): 58-73.

Iera, Antonio, Antonella Molinaro, and Salvatore Marano. 2002. “Traffic Management Techniques to Face the Effects of Intrinsic Delays in Geostationary Satellite Networks." IEEE Transactions on Wireless Communications 1 (1): 145-55. https:// doi.org/10.1109/7693.975453.

Kassim, M., S.F. Ramle, R.A. Rahman, and M.I. Yusof. 2017. “Skype Multimedia Application Traffic Analysis on Home Unifi Network.” In ISCAIE 2017 - 2017 IEEE Symposium on Computer Applications and Industrial Electronics, 184-89. https://doi.org/10.1109/ISCAIE.2017.8074974.

Lester, Dan, and Harley Thronson. 2011. "Human Space Exploration and Human Spaceflight: Latency and the Cognitive Scale of the Universe.” Space Policy 27 (2): 89-93. https://doi.org/10.1016/j.spacepol.2011.02.002.

Love, Stanley G., and Marcum L. Reagan. 2013. "Delayed Voice Communication.” Acta Astronautica 91: 89-95. https://doi.org/10.1016/j.actaastro.2013.05.003.

Lum, M J H, J Rosen, T S Lendvay, M N Sinanan, B Hannaford, and Ieee. 2009. “Effect of Time Delay on Tele Surgical Performance.” Icra: 2009 Ieee International Conference on Robotics and Automation, Vols 1-7, 3447-53. https://doi.org/10.1109/ ROBOT.2009.5152725.

Sanctis, Mauro De, Ernestina Cianca, Giuseppe Araniti, Igor Bisio, and Ramjee Prasad. 2016. “Satellite Communications Supporting Internet of Remote Things.” IEEE Internet of Things Journal 3 (1): 113-23.

Santos, João, João Pedro, Antonio Eira, Paulo Monteiro, and João Pires. 2012. "Optical Transport Network Design with Collocated Regeneration and Differential Delay Compensation.” 2012 IEEE 13th International Conference on High 
Performance Switching and Routing, HPSR 2012, 204-9. https://doi.org/10.1109/ HPSR.2012.6260851.

Sarkar, Mohanchur. 2011. "A Survey of Transport Protocols for Deep Space Communication Networks." International Journal of Computer Applications 31 (8): 25-32.

Schalk, Kevin, Kai Muller, Karsten Peter, and Johannes Sebald. 2017. “Microsecond-Precision Time Stamping in a Deterministic Distributed Sensor Network Utilizing OpenPOWERLINK." 2017 IEEE International Conference on Wireless for Space and Extreme Environments (WiSEE), 52-56. https://doi.org/10.1109/ WiSEE.2017.8124892.

$\mathrm{Su}$, Chien-chung, Ko-ming Chang, Yau-hwang Kuo, and a Symmetric Key Management. 2005. “The New Intrusion Prevention and Detection Approaches for Clustering-Based Sensor Networks.” In IEEE Wireless Communications and Networking Conference, 1927-32. https://doi.org/10.1109/WCNC.2005.1424814.

Theodore Holmes Bullock. 1973. “Seeing the World through a New Sense: Electroreception in Fish: Sharks, Catfish, and Electric Fish Use Low- or High-Frequency Electroreceptors, Actively and Passively, in Object Detection and Social Communication.” American Scientist 61 (3): 316-25.

Yunlong Feng Gene Cheung, Wai-tian T A N. 2013. "Low-Cost Eye Gaze Prediction System for Intereactive Networked Video Streaming.” IEEE Transanction On Multimedia 15 (8): 1865-79.

\section{Acknowledgements}

Special thanks to Javier S. Cañizares and Ignacio R. Matias for their support and assistance in writing this manuscript. 